International Journal of Pure and Applied Mathematics

Volume 110 No. 2 2016, 257-264

ISSN: 1311-8080 (printed version); ISSN: 1314-3395 (on-line version)

url: http://www.ijpam.eu

doi: 10.12732 /ijpam.v110i2.2

\title{
A NEW SUBCLASS OF P-VALENT FUNCTIONS DEFINED BY AOUF ET AL DERIVATIVE OPERATOR
}

\author{
Jamiu Olusegun Hamzat ${ }^{1}$, Olawale Joshua Adeleke ${ }^{2} \S$ \\ ${ }^{1}$ Department of Pure and Applied Mathematics \\ LAUTECH, PMB 4000, Ogbomoso, NIGERIA \\ ${ }^{2}$ Department of Mathematics \\ Covenant University \\ Ota, NIGERIA
}

\begin{abstract}
In this present paper, we study a new subclass of $\delta_{p}(A, B, b, \omega, \lambda, l, n)$ of analytic $p$-valent functions in the unit disk $U=\{z:|z|<1\}$ defined by Aouf et al derivative operator. Coefficient estimates and relevant connection of this class to the famous Fekete-Szego were obtained.
\end{abstract}

AMS Subject Classification: 30C45

Key Words: analytic function, univalent function, starlike function, convex function, coefficient inequalities

\section{Introduction}

Let $S(\omega)$ be the class of functions $f(z)$ of the form

$$
\begin{aligned}
f(z) & =(z-\omega)+\sum_{k=2}^{\infty} a_{k}(z-\omega)^{k}, \\
U & =\{z: z \in C \text { and }|z|<1\},
\end{aligned}
$$

which are analytic and univalent in the open unit disk $\mathrm{U}$, normalized with

Received: January 20, 2016

Revised: June 20, 2016

Published: November 4, 2016

$\S_{\text {Correspondence author }}$ (c) 2016 Academic Publications, Ltd.

url: www.acadpubl.eu 
$f(\omega)=f^{\prime}(\omega)-1=0$ and $\omega$ is an arbitrary fixed point in $U$.

Also, let $\delta_{p}(\omega)$ denote the class of analytic p-valent functions of the form:

$$
h(z)=(z-\omega)^{p}+\sum_{k=1}^{\infty} a_{p+k}(z-\omega)^{p+k},
$$

defined in the unit disk where $\omega$ is an arbitrary fixed point in $U$ and satisfying the conditions $h(\omega)=0,|h(z)|<1, z \in U$.

Wald in [11] established that, if $P(\omega) \subset P$ (i.e class of caratheodary functions) and $p(z)$ is of the form

$$
p(z)=1+\sum_{n=1}^{\infty} p_{k}(z-\omega)^{k}
$$

then

$$
\left|p_{k}\right| \leq \frac{2}{(1+d)(1-d)^{k}}, \quad k \geq 1 \text { and }|\omega|=d
$$

This result is scattered in many literatures $[1,2,5,6,7,8]$ and we also note that if $A$ and $B$ are arbitrarily fixed integers and $-1 \leq B<A \leq 1$, then we have

$$
\left|p_{k}\right| \leq \frac{A-B}{(1+d)(1-d)^{k}}, \quad k \geq 1,-1 \leq B<A \leq 1, \text { and }|\omega|=d .
$$

For $f(z) \in \delta_{p}(\omega)$, Eker and Seker in [9] introduced the following differential operator

$$
\begin{aligned}
& D^{0} f(z)=f(z), \\
& D^{1} f(z)=D(f(z))=\frac{(z-\omega)}{p} f^{\prime}(z)=(z-\omega)^{p}+\sum_{k=1}^{\infty}\left(\frac{p+k}{p}\right) a_{p+k}(z-\omega)^{p+k}, \\
& D^{2} f(z)=\frac{(z-\omega)}{p} f^{\prime}(z)=(z-\omega)^{p}+\sum_{k=1}^{\infty}\left(\frac{p+k}{p}\right)^{2} a_{p+k}(z-\omega)^{p+k},
\end{aligned}
$$

and

$$
\begin{array}{r}
D^{n+p} f(z)=D\left(D^{n+p-1} f(z)\right)=(z-\omega)^{p}+\sum_{k=1}^{\infty}\left(\frac{p+k}{p}\right)^{n} a_{p+k}(z-\omega)^{p+k} \\
m \in N_{0} .
\end{array}
$$


Using Aouf et al derivative operator (see [3]), we can write for functions $f(z) \in \delta_{p}(\omega):$

$$
\begin{aligned}
I_{\omega, p}^{0}(\lambda, l) f(z)= & f(z), \\
I_{\omega, p}^{1}(\lambda, l) f(z)= & \left(I_{\omega, p}^{0}(\lambda, l) f(z)\right)\left(\frac{1-\lambda+l}{1+l}\right)+\left(I_{\omega, p}^{0}(\lambda, l) f(z)\right)^{\prime} \frac{\lambda z}{1+l} \\
= & \left(\frac{1+\lambda(p-1)+l}{1+l}\right)(z-\omega)^{p} \\
& +\sum_{k=1}^{\infty}\left(\frac{1+\lambda(p+k-1)+l}{1+l}\right) a_{p+k}(z-\omega)^{p+k}, \\
I_{\omega, p}^{2}(\lambda, l) f(z)= & \left(\frac{1+\lambda(p-1)+l}{1+l}\right)(z-\omega)^{p} \\
& +\sum_{k=1}^{\infty}\left(\frac{1+\lambda(p+k-1)+l}{1+l}\right)^{2} a_{p+k}(z-\omega)^{p+k},
\end{aligned}
$$

and in general, we have

$$
\begin{aligned}
& I_{\omega, p}^{n}(\lambda, l) f(z)=\left(\frac{1+\lambda(p-1)+l}{1+l}\right)^{n}(z-\omega)^{p} \\
& +\sum_{k=1}^{\infty}\left(\frac{1+\lambda(p+k-1)+l}{1+l}\right)^{n} a_{p+k}(z-\omega)^{p+k}, \quad n \in N_{0}, \quad \lambda \geq 0, \quad l \geq 0 .
\end{aligned}
$$

With $\omega=0, l=0$ and $\lambda=1$. It is trivial to show that

$$
\frac{1}{p^{n}} I_{0, p}^{n}(1,0) f(z)=D^{n+p} f(z) .
$$

Now, the authors wish to introduce the class $\delta_{p}(A, B, b, \omega, \lambda, l, n)$ consisting of functions $f(z)$ of the form (1) and satisfying

$$
\begin{aligned}
& 1+\frac{1}{b}\left\{\frac{(z-\omega)\left(\frac{1}{p^{n}} I_{\omega, p}^{n}(\lambda, l) f(z)\right)^{\prime}}{\left(\frac{1}{p^{n}} I_{\omega, p}^{n}(\lambda, l) f(z)\right)}-p\right\} \prec \frac{1+A(z-\omega)}{1+B(z-\omega)} \\
& -1 \leq B<A \leq 1, n \in N_{0}, \lambda \geq 0, l \geq 0, z \in U,
\end{aligned}
$$

where $\mathrm{b}$ is any non-zero complex number, $\prec$ denotes subordination and $\omega$ is an arbitrary fixed point in $U$. With various choices of parameters involved, this 
class of functions would give birth to several existing ones by several authors, see $[1],[3],[4]$.

Hence, by the definition of subordination it follows that $f \in \delta_{p}(A, B, b, \omega, \lambda$, $l, n)$ if and only if

$$
1+\frac{1}{b}\left\{\frac{(z-\omega)\left(\frac{1}{p^{n}} I_{\omega, p}^{n}(\lambda, l) f(z)\right)^{\prime}}{\left(\frac{1}{p^{n}} I_{\omega, p}^{n}(\lambda, l) f(z)\right)}-p\right\}=\frac{1+A h(z-\omega)}{1+B h(z-\omega)}=p(z)
$$

$z, h \in U$,

where $p(z)$ is as earlier defined in $(2)$.

\section{Main Results}

In this section, we give the coefficient estimates for the classes $\delta_{p}(A, B, b, \omega$, $\lambda, l, n)$.

Theorem 1. Let $f(z) \in \delta_{p}(A, B, b, \omega, \lambda, l, n)$, then for $k \geq 1, \lambda \geq 0, l \geq 0$, $-1 \leq B<A \leq 1, p \in N$ and $z \in U$ :

$$
\begin{aligned}
\left|a_{2}\right| \leq & \frac{|b|(A-B)}{\left(1-d^{2}\right)\left(\frac{1+\lambda+l}{1+l}\right)^{n}}, \\
\left|a_{3}\right| \leq & \frac{|b|^{2}(A-B)^{2}+|b|(A-B)(1+d)}{2\left(1-d^{2}\right)^{2}\left(\frac{1+2 \lambda+l}{1+l}\right)^{n}}, \\
\left|a_{4}\right| \leq & \frac{|b|^{3}(A-B)^{3}+3|b|^{2}(A-B)^{2}(1+d)+2|b|(A-B)(1+d)}{2.3\left(1-d^{2}\right)^{3}\left(\frac{1+3 \lambda+l}{1+l}\right)^{n}}, \\
\left|a_{5}\right| \leq & \left(|b|^{4}(A-B)^{4}+4|b|^{3}(A-B)^{3}(1+d)+4|b|^{2}(A-B)^{2}(1+d)^{2}\right. \\
& \left.+|b|(A-B)(1+d)^{3}\right)\left(2.3 .4\left(1-d^{2}\right)^{4}\left(\frac{1+4 \lambda+l}{1+l}\right)^{n}\right)^{-1} .
\end{aligned}
$$

Proof. From (5)

$$
1+\frac{1}{b}\left\{\frac{(z-\omega)\left(\frac{1}{p^{n}} I_{\omega, p}^{n}(\lambda, l) f(z)\right)^{\prime}}{\left(\frac{1}{p^{n}} I_{\omega, p}^{n}(\lambda, l) f(z)\right)}-p\right\}=1+\sum_{k=1}^{\infty} p_{k}(z-\omega)^{k} .
$$


That is

$$
\left\{\frac{(z-\omega)\left(\frac{1}{p^{n}} I_{\omega, p}^{n}(\lambda, l) f(z)\right)^{\prime}}{\left(\frac{1}{p^{n}} I_{\omega, p}^{n}(\lambda, l) f(z)\right)}-p\right\}=\sum_{k=1}^{\infty} b p_{k}(z-\omega)^{k} .
$$

Using (4) on (6), we have

$$
\begin{aligned}
& \sum_{k=1}^{\infty} \frac{k}{p^{n}}\left(\frac{1+\lambda(p+k-1)+l}{1+l}\right)^{n} a_{p+k}(z-\omega)^{p+k} \\
& \overline{\frac{1}{p^{n}}\left[\left(\frac{1+\lambda(p-1)+l}{1+l}\right)^{n}(z-\omega)^{p}+\sum_{k=1}^{\infty}\left(\frac{1+\lambda(p+k-1)+l}{1+l}\right)^{n} a_{p+k}(z-\omega)^{p+k}\right]} \\
& =\sum_{k=1}^{\infty} b p_{k}(z-\omega)^{k}
\end{aligned}
$$

Dividing the numerator denominator of the L.H.S. by the factor $(z-\omega)^{p}$, then we have

$$
\begin{gathered}
\frac{\sum_{k=1}^{\infty} \frac{k}{p^{n}}\left(\frac{1+\lambda(p+k-1)+l}{1+l}\right)^{n} a_{p+k}(z-\omega)^{k}}{\frac{1}{p^{n}}\left[\left(\frac{1+\lambda(p-1)+l}{1+l}\right)^{n}+\sum_{k=1}^{\infty}\left(\frac{1+\lambda(p+k-1)+l}{1+l}\right)^{n} a_{p+k}(z-\omega)^{k}\right]} \\
=\sum_{k=1}^{\infty} b p_{k}(z-\omega)^{k} .
\end{gathered}
$$

By expanding (7) and equating the coefficient of the like powers, we have

$$
\begin{aligned}
\left(\frac{1+\lambda p+l}{1+l}\right)^{n} a_{p+1}= & b\left(\frac{1+\lambda(p-1)+l}{1+l}\right)^{n} p_{1} \\
2\left(\frac{1+\lambda(p+1)+l}{1+l}\right)^{n} a_{p+2}= & b\left(\frac{1+\lambda p+l}{1+l}\right)^{n} a_{p+1} p_{1}+b\left(\frac{1+\lambda(p-1)+l}{1+l}\right)^{n} p_{2} \\
3\left(\frac{1+\lambda(p+2)+l}{1+l}\right)^{n} a_{p+3}= & b\left(\frac{1+\lambda(p+1)+l}{1+l}\right)^{n} a_{p+2} p_{1} \\
& +b\left(\frac{1+\lambda p+l}{1+l}\right)^{n} a_{p+1} p_{2} \\
& +b\left(\frac{1+\lambda(p-1)+l}{1+l}\right)^{n} p_{3} \\
4\left(\frac{1+\lambda(p+3)+l}{1+l}\right)^{n} a_{p+4}= & b\left(\frac{1+\lambda(p+2)+l}{1+l}\right)^{n} a_{p+3} p_{1}
\end{aligned}
$$




$$
\begin{aligned}
& +b\left(\frac{1+\lambda(p+1)+l}{1+l}\right)^{n} a_{p+2} p_{2} \\
& +b\left(\frac{1+\lambda p+l}{1+l}\right)^{n} a_{p+1} p_{3} \\
& +b\left(\frac{1+\lambda(p-1)+l}{1+l}\right)^{n} p_{4} .
\end{aligned}
$$

Now, if we let $p=1$ and using (3) on the above then

$$
\begin{aligned}
\left|a_{2}\right| \leq & \frac{|b|(A-B)}{\left(1-d^{2}\right)\left(\frac{1+\lambda+l}{1+l}\right)^{n}}, \\
\left|a_{3}\right| \leq & \frac{|b|^{2}(A-B)^{2}+|b|(A-B)(1+d)}{2\left(1-d^{2}\right)\left(\frac{1+2 \lambda+l}{1+l}\right)^{n}}, \\
\left|a_{4}\right| \leq & \frac{|b|^{3}(A-B)^{3}+3|b|^{2}(A-B)^{2}(1+d)+2|b|(A-B)(1+d)^{2}}{2.3\left(1-d^{2}\right)^{3}\left(\frac{1+3 \lambda+l}{1+l}\right)^{n}}, \\
\left|a_{5}\right| \leq & \left(|b|^{4}(A-B)^{4}+4|b|^{3}(A-B)^{3}(1+d)+4|b|^{2}(A-B)^{2}(1+d)^{2}\right. \\
& \left.+|b|(A-B)(1+d)^{3}\right)\left(2.3 .4\left(1-d^{2}\right)^{4}\left(\frac{1+4 \lambda+l}{1+l}\right)^{n}\right)^{-1} .
\end{aligned}
$$

This complete the proof of Theorem 2.1.

If $n=0$ in Theorem 2.1, above, then we have for $k \geq 1$ and $p=1$.

Corollary 2. Let $f \in \delta_{1}(A, B, b, \omega, \lambda, l, 0)$ then:

$$
\begin{aligned}
\left|a_{2}\right| \leq & \frac{|b|(A-B)}{\left(1-d^{2}\right)}, \\
\left|a_{3}\right| \leq & \frac{|b|^{2}(A-B)^{2}+|b|(A-B)(1+d)}{2\left(1-d^{2}\right)}, \\
\left|a_{4}\right| \leq & \frac{|b|^{3}(A-B)^{3}+3|b|^{2}(A-B)^{2}(1+d)+2|b|(A-B)(1+d)^{2}}{2.3\left(1-d^{2}\right)^{3}}, \\
\left|a_{5}\right| \leq & \left(|b|^{4}(A-B)^{4}+4|b|^{3}(A-B)^{3}(1+d)+4|b|^{2}(A-B)^{2}(1+d)^{2}\right. \\
& \left.+|b|(A-B)(1+d)^{3}\right)\left(2.3 .4\left(1-d^{2}\right)^{4}\right)^{-1} \cdot
\end{aligned}
$$


Also, setting $n=d=0$ and $p=1$ in Theorem 1, we receive.

Corollary 3. Let $f \in \delta_{1}(A, B, b, \omega, \lambda, l, 0)$, then

$$
\begin{aligned}
& \left|a_{2}\right| \leq|b|(A-B), \\
& \left|a_{3}\right| \leq \frac{|b|(A-B)[1+|b|(A-B)]}{2}, \\
& \left|a_{4}\right| \leq \frac{|b|(A-B)\left[2+3|b|(A-B)+|b|^{2}(A-B)^{2}\right]}{2.3}, \\
& \left|a_{5}\right| \leq \frac{|b|(A-B)\left[1+4|b|(A-B)+4|b|^{2}(A-B)^{2}+|b|^{3}(A-B)^{3}\right]}{2.3 .4} .
\end{aligned}
$$

With several other choices of the parameters involved both the existing and many new coefficient estimate could be obtained.

Our next result shows the relevance of our class $\delta_{1}(A, B, b, \omega, \lambda, l, 0)$ to the famous Fekete-Zsego Theorem.

Theorem 4. Let $f \in \delta_{1}(A, B, b, \omega, \lambda, l, n)$, then

$$
\begin{aligned}
& \left|a_{3}-\mu a_{2}^{2}\right| \leq \\
& \frac{|b|(A-B)\left[|b|(A-B)+(1+d)\left(\frac{1+\lambda+l}{1+l}\right)^{n}-2 \mu|b|(A-B)\left(\frac{1+2 \lambda+l}{1+l}\right)^{n}\right]}{2\left(1-d^{2}\right)^{2}\left(\frac{1+2 \lambda+l}{1+l}\right)^{n}\left(\frac{1+\lambda+l}{1+l}\right)^{2 n}} .
\end{aligned}
$$

Proof. The proof follows from Theorem 2.1. With various choices of parameters involved, several connections of our class to the well known Fekete-Zsego Theorem could be obtained.

If $n=0$ in Theorem 4, we have the following corollary.

Corollary 5. Let $f \in \delta_{1}(A, B, b, \omega, \lambda, l, 0)$, then

$$
\left|a_{3}-\mu a_{2}^{2}\right| \leq \frac{|b|(A-B)[|b|(A-B)+(1+d)-2 \mu|b|(A-B)]}{2\left(1-d^{2}\right)^{2}} .
$$

Setting $n=d=0$, we have

Corollary 6. Let $f \in \delta_{1}(A, B, b, \omega, \lambda, l, 0)$, then

$$
\left|a_{3}-\mu a_{2}^{2}\right| \leq \frac{|b|(A-B)[1+|b|(A-B)-2 \mu|b|(A-B)]}{2} .
$$


Also, if $n=\lambda=1$, and $l=0$, we have

Corollary 7. Let $f \in \delta_{1}(A, B, b, \omega, 1,0,1)$

$$
\left|a_{3}-\mu a_{2}^{2}\right| \leq \frac{|b|(A-B)[|b|(A-B)+2(1+d)-6 \mu|b|(A-B)]}{2.2 .3(1-d)^{2}} .
$$

Setting $n=\lambda=1$ and $l=d=0$, we obtain

Corollary 8. Let $f \in \delta_{1}(A, B, b, \omega, 1,0,1)$

$$
\left|a_{3}-\mu a_{2}^{2}\right| \leq \frac{|b|(A-B)[2+|b|(A-B)-6 \mu|b|(A-B)]}{2.2 .3} .
$$

\section{References}

[1] A. Akbarally, S. Cik Sohb and M. Ismail, On the properties of a class of $p$-valent functions defined by salagean differential operator, Int. Journal of Math. Analysis, 5, No. 21 (2011), 1035-1045.

[2] M. Acu and S.Owa, On some subclasses of univalent functions, Journal of Inequalities in Pure and Applied Mathematics, 6, No. 3, Article 70 (2005), 1-14.

[3] M.K Aouf, A.Shamandy, A.O Mostafa, and S.M Madian, A subclass of M-W starlike functions, Acta Universitatis Apulensis, No. 21 (2010), 135-142.

[4] R.M. Goel and B.C. Mehok, A subclass of starlike functions with respect to symmetric points, Tamkang J. Math., 13, No. 1 (1982), 11-24.

[5] S.Kanas, and F.Ronning, Uniformly starlike and convex functions and other related classes of univalent functions, Ann. Univ. Mariae Curie-Sklodowska section A, 53 (1999), 95-105.

[6] A.T. Oladipo, On subclasses of univalent functions, Advances in Applied Mathematical Analysis, 4, No. 2 (2009), 87-93.

[7] A. T. Oladipo and D. Breaz, On the family of Bazilevic functions, Acta Universitatis Apulensis, No. 24 (2004).

[8] A. T. Oladipo and S.O. Olatunji, On new subclasses of analytic functions with respect to other points defined by aouf et al. operator, ICASTOR Journal of Mathematical Sciences, 5, No. 2 (2011) 245-253.

[9] S.S. Seker and B. Eker, On a class of multivalent functions defined by Salagean Operator, General Mathematics, 15 (2007), 154-163.

[10] C. Selvaraj and N. Vasanthi, Subclasses of analytic functions with respect to symmetric and conjugate points, Tamkang Journal of Mathematics, 42, No. 1 (2011), 87-94.

[11] J. K Wald, On Starlike Functions, Ph.D. Thesis, University of Delaware, New Ark, Delaware (1978). 